\title{
COMMUNICATION
}

\section{À propos d'un cas de nécrose osseuse mandibulaire : ostéite chronique suppurée ou ostéochimionécrose ?}

\author{
Renoux M ${ }^{1,2}$, Pajot $\mathrm{T}^{2,3}$, Radoï $\mathrm{L}^{1,2}$. \\ 1 - Faculté de chirurgie dentaire Paris Descartes, département de médecine buccale et de chirurgie buccale, \\ Montrouge, France
}

2 - Hôpital Louis Mourier, service d'odontologie (AP-HP), Colombes, France

3 - Interne en DESCO

\section{Introduction}

Les ostéites maxillo-faciales ont généralement une cause locale et, plus rarement, une cause locorégionale ou générale. Les signes cliniques associent des douleurs, une tuméfaction, des adénopathies et, plus tardivement, une séquestration osseuse. L'imagerie (orthopantomogramme, TDM, IRM, scintigraphie) permet de préciser l'ampleur de l'atteinte osseuse et des tissus mous et de suivre l'évolution des lésions dont le traitement est de longue durée'.

\section{Observation}

Un patient de 86 ans a consulté pour une tuméfaction mandibulaire d'apparition récente. Parmi ses antécédents médicaux ont été retrouvés : le remplacement récent de sa prothèse totale de hanche droite avec plusieurs complications au cours de l'hospitalisation (notamment une septicémie), une hypertension artérielle stabilisée et une maladie de Horton traitée par corticothérapie, un traitement par alendronate (70 mg/semaine pendant 13 mois), arrêté 6 mois auparavant du fait d'une mauvaise tolérance digestive. L'examen exo-buccal montrait une tuméfaction génienne basse gauche, douloureuse, recouverte d'une peau érythémateuse. L'examen endo-buccal montrait un édentement mandibulaire total. II existait une déformation de la table osseuse externe mandibulaire, douloureuse à la palpation, et deux fistules productives. L'orthopantomogramme faisait suspecter une image ostéolytique mal délimitée au niveau du corps mandibulaire gauche, justifiant la prescription d'une TDM. Une antibiothérapie probabiliste a été instaurée (amoxicilline 2g/j, métronidazole 1,5g/j). La TDM n'a pas permis d'objectiver clairement l'ostéolyse. Une scintigraphie osseuse, examen plus sensible et plus précoce que la TDM, a été prescrite ; elle a révélé un foyer unique d'hyperfixation mandibulaire gauche. Deux curetages consécutifs ont été nécessaires du fait de la persistance des fistules puis de l'apparition d'une exposition et d'une séquestration osseuse. Les examens anatomopathologiques ont conduit au diagnostic d'ostéite suppurée. La première analyse microbiologique a révélé la présence d'entérobactéries non commensales de la cavité buccale, Enterococcus gallinarum et Escherichia coli, résistantes au traitement prescrit, nécessitant sa modification (ofloxacine $800 \mathrm{mg} / \mathrm{j}$, cefoxitine $400 \mathrm{mg} / \mathrm{j}$ ). La seconde étude microbiologique a révélé la présence d'une autre entérobactérie pathogène opportuniste, Morganella morganii, multi-résistante. L'antibiothérapie a été réadaptée (céfépime injectable $2 \mathrm{~g} / \mathrm{j}$ ). A 1 mois, la cicatrisation muqueuse était complète. L'orthopantomogramme montrait une cicatrisation osseuse en cours à 3 mois.

This is an Open Access article distributed under the terms of the Creative Commons Attribution License 4.0, which permits unrestricted use, distribution, and reproduction in any medium, provided the original work is properly cited. 
Discussion

Les deux principales hypothèses diagnostiques étaient une ostéite mandibulaire chronique suppurée ou une ostéochimionécrose de stade 4. Les ostéites maxillo-faciales sont des affections rares, favorisées par certains facteurs : âges extrêmes, immunodéficience, malnutrition, ostéopathies... ${ }^{1}$ Les entérobactéries non commensales de la cavité buccale sont pathogènes, soit strictes, soit opportunistes. Ces dernières sont responsables de septicémies et d'infections à distance dans des contextes particuliers (immunodépression, milieu hospitalier, intervention chirurgicale) $)^{2}$. L'ostéochimionécrose, surinfectée par des germes d'origine entérale, chez un patient dépendant, était moins plausible du fait de la durée courte du traitement par alendronate, prescrit pour ostéoporose. Les entérobactéries pathogènes sont multi-résistantes aux antibiotiques prescrits usuellement en odontostomatologie ${ }^{3}$, ce qui impose de réaliser des antibiogrammes afin d'instaurer un traitement efficace.

\section{Conclusion}

Le traitement des ostéites chroniques suppurées comporte un geste chirurgical et une antibiothérapie de longue durée, adaptée au germe causal. La réévaluation de l'efficacité du traitement inclut plusieurs examens microbiologiques afin de suivre l'évolution de la population bactérienne et d'adapter l'antibiothérapie aux résistances acquises.

\section{Références}

1- Maes JM et al. Ostéites des os de la face. EMC, Paris (Elsevier Masson SAS), Médecine Buccale, 28-405-M-10, 2008.

2- Sabders WE Jr et al. Enterobacter spp.: pathogens poised to flourish at the turn of the century. Clin Microbiol Rev $1997 ; 10: 220-41$.

3- Sougakoff V et al. Résistances aux beta-lactamines. Service de Batériologie-Hygiène-Pitié-Salpêtrière, Paris, 2003 ; http://www.chups.jussieu.fr/polys/bacterio/resistlacta/ index.html, consulté le 01/11/2015. 